\title{
Hugoniot States and Mie-Grüneisen Equation of State of Iron Estimated Using Molecular Dynamics
}

\author{
Yuntian Wang ${ }^{1}$, Xiangguo Zeng ${ }^{1, *}$, Huayan Chen ${ }^{1, *}$, Xin Yang ${ }^{2}$, Fang Wang ${ }^{3}$ and Jun Ding 4 \\ 1 MOE Key Laboratory of Deep Earth Science and Engineering, College of Architecture and Environment, \\ Sichuan University, Chengdu 610065, China; iswangyt@163.com \\ 2 State Key Laboratory of Geohazard Prevention and Geoenvironment Protection, \\ Chengdu University of Technology, Chengdu 610059, China; scsnyangxin@sina.com \\ 3 School of Materials and Energy, Southwest University, Chongqing 400715, China; wangfang_cq1978@163.com \\ 4 College of Mechanical Engineering, Chongqing University of Technology, Chongqing 400054, China; \\ dingjun@cqut.edu.cn \\ * Correspondence: xiangguozeng@scu.edu.cn (X.Z.); kay_chy@126.com (H.C.)
}

check for updates

Citation: Wang, Y.; Zeng, X.; Chen, H.; Yang, X.; Wang, F.; Ding, J.

Hugoniot States and Mie-Grüneisen Equation of State of Iron Estimated Using Molecular Dynamics. Crystals 2021, 11, 664. https://doi.org/ $10.3390 /$ cryst11060664

Academic Editor: Francesco Stellato

Received: 21 April 2021

Accepted: 8 June 2021

Published: 10 June 2021

Publisher's Note: MDPI stays neutral with regard to jurisdictional claims in published maps and institutional affiliations.

Copyright: (c) 2021 by the authors. Licensee MDPI, Basel, Switzerland. This article is an open access article distributed under the terms and conditions of the Creative Commons Attribution (CC BY) license (https:// creativecommons.org/licenses/by/ $4.0 /)$.

\begin{abstract}
The objective of this study was to develop a micromechanical approach for determining the Mie-Grüneisen EOS parameters of iron under the Hugoniot states. The multiscale shock technique (MSST) coupled with molecular dynamics (MD) simulations was employed to describe the shocked Hugoniot relation of single-crystal (SC) and nanocrystalline (NC) iron under high pressures. The Mie-Grüneisen equation of state (EOS) parameters, the cold pressure $\left(P_{c}\right)$, the cold energy $\left(E_{c}\right)$, the Grüneisen coefficient $(\gamma)$, and the melting temperature $\left(T_{m}\right)$ are discussed. The error between SC and $\mathrm{NC}$ iron results was found to be less than $1.5 \%$. Interestingly, the differences in Hugoniot state $\left(P_{H}\right)$ and the internal energy between SC and NC iron were insignificant, which shows that the effect of grain size (GS) under high pressures was not significant. The $P_{\mathcal{C}}$ and $E_{\mathcal{C}}$ of SC and NC iron calculated based on the Morse potential were almost the same with those calculated based on the Born-Mayer potential; however, those calculated based on the Born-Mayer potential were a little larger at high pressures. In addition, several empirical and theoretical models were compared for the calculation of $\gamma$ and $T_{m}$. The Mie-Grüneisen EOSs were shown on the 3D contour space; the pressure obtained with the Hugoniot curves as the reference was larger than that obtained with the cold curves as the reference.
\end{abstract}

Keywords: multiscale shock technique; iron; hugoniot states; Mie-Grüneisen equation of state parameters; molecular dynamics

\section{Introduction}

Iron is a major element in the Earth's core. The properties of iron at elevated temperatures and pressures are crucial to model the temperature profile and the dynamics of the earth core, where pressures could reach up to 130-370 GPa [1,2]. Under high-pressure conditions, the behavior of metallic materials can be described by the equation of state (EOS) [3]. In the study on high-pressure physics, a deep understanding of EOS and other characteristics, such as the shock Hugoniot, Grüneisen coefficient $(\gamma)$, and melting temperature $\left(T_{m}\right)$, is vital [4,5].

The shock Hugoniot relations, which can be applied to calculate the EOS, have received great attention [6,7]. Barker [8] reexamined the $\alpha$-iron Hugoniot at around $13 \mathrm{GPa}$. The results showed that the Hugoniot $\alpha$-phase iron was quite normal under $13 \mathrm{GPa}$, and there was no indication of an early phase transition to $\epsilon$-iron. Thomas et al. [9] investigated the shock Hugoniot of $\alpha$-iron under 10 GPa through low-velocity symmetric impact experiments. The results provided reliable data and fit for $\alpha$-iron under low-stress conditions below $10 \mathrm{GPa}$. Above $13 \mathrm{GPa}$, a solid-solid phase $(\alpha-\epsilon)$ transition will occur in iron [10]. Brown et al. [11] collected the shock Hugoniot data of iron $(<0.3 \%$ carbon $)$ in Los Alamos 
from about 39 to $442 \mathrm{GPa}$, which were obtained using explosive driven shock and a twostage light-gas gun system. Zaretsky [12] studied the shock response of polycrystalline iron using the planar impact experiment method and determined the borders of stability of iron $\alpha$ - and $\gamma$-phases. Liu et al. [13] investigated the crystal orientation effect on the phase transition stress of single iron under shock compression; the results show that the orientation effect on the phase transition stresses of single crystal iron is not obvious. Sjostrom and Crockett [14] conducted quantum molecular dynamics simulation to investigate the shock Hugoniot and equation of state of warm dense iron; the shock Hugoniot of up to $20 \mathrm{TPa}$ agrees with the experimental results. Kadau et al. $[15,16]$ investigated the shock-induced phase transformations in single-crystal (SC) and polycrystalline iron using nonequilibrium molecular dynamics (NEMD) simulations. The dynamics of shock-induced transformations were discussed, and the Hugoniot data of SC and polycrystalline iron under pressures up to $300 \mathrm{GPa}$ were obtained.

The Mie-Grüneisen EOS, which relates the pressure $(P)$, specific volume $(V)$, and internal energy $(E)$, is widely used to characterize the behavior of metal materials in highpressure physics [17-23]. In the study by Heuzé [24], the general $P(V, E)$ Mie-Grüneisen form was extended to a complete $S(V, E)$ form, which could provide all of the thermodynamic properties. Zhang et al. [25] proposed a cold energy mixture theory in which the Mie-Grüneisen EOS is calculated from the cold curves and Hugoniot curves, and the authors also examined $\gamma$. In the Mie-Grüneisen EOS, $\gamma$ plays an important role, as it can clarify the melting of solids at extreme conditions [26]. There are three models for characterizing the Grüneisen coefficient $\gamma$, the Slater model [27], the Dugdale-MacDonald model [28], and the free-volume model [29]. In early 1963, Vashchenko and Zubarev [29] proposed the idea of writing a unified equation for the three models. Based on this, Al'tshuler [30] gives a unified expression of the Grüneisen coefficient $\gamma$. Cui and $\mathrm{Yu}$ [31] proposed a model to calculate $\gamma$ at high temperatures. The model agrees well with the experimental data of iron in a wide range of pressures. Sha and Cohen [32] used first-principle calculations to study the $\gamma$ of bcc iron under pressures up to $40 \mathrm{GPa}$ and temperature of $2000 \mathrm{~K}$. The calculated $\gamma$ showed good agreement with the experiments, and the variation of $\gamma$ with temperature was remarkable, but the variation with pressure was moderate. Li et al. [33] calculated the $\gamma$ of iron in the pressure range of $90-160 \mathrm{GPa}$ based on the Hugoniot data obtained by plate impact experiments. The results show that $\gamma_{0} \cdot \rho_{0}=\gamma \cdot \rho=$ const in the pressure range.

Shock-induced melting is an important phenomenon for studying the solid-liquid phase transition and the nature of material melting at extreme conditions, and the melting temperature $T_{m}$ is related to $\gamma_{0}$. Based on the Lindemann melting criterion and the volume dependence of the Gruneisen coefficient $\gamma$, Yang et al. [5] investigated the melting characteristics of single and polycrystalline Al. Errandonea [34] used a laser-heated diamond-anvil cell to study the melting characteristics of $\mathrm{Cu}, \mathrm{Ni}, \mathrm{Pd}$, and $\mathrm{Pt}$ in high-pressure conditions; it was shown that Simon equations could be used to describe the melting curves. Anzellini et al. [35] conducted static laser-heated diamond anvil cell experiments to investigate the melting point of iron under pressures up to $200 \mathrm{GPa}$. The results indicated that the temperature of iron at the inner core boundary was $6230 \pm 500 \mathrm{~K}$. Bouchet et al. [36] calculated the melting curve, and EOS of hcp and bcc iron under pressures up to $1500 \mathrm{GPa}$; the melting temperature was $11,000 \mathrm{~K}$ at the highest pressure.

MD simulation has become an important research method in high-pressure physics. For example, the NEMD method [37], equilibrium molecular dynamics (EMD) method, [38] and first-principle method [39] have been successfully applied to study the shock Hugoniot, $\gamma$, and $T_{m}$. The time scale and model size in NEMD and EMD simulations are restricted by computing resources; as the time scale and model size increase, the computational time will significantly increase. The first-principle method can fundamentally calculate the molecular structure and material properties, but the model size is too small in the shockwave propagation simulation. To solve the computational time and model size shortcomings in the above methods, Reed et al. [40] proposed a multiscale simulation method, called the multiscale shock technique (MSST), to study the shockwave propagation 
in materials. Compared with the above methods, the MSST method can efficiently save computational time and guarantee simulation accuracy with an acceptable model size of the MD system [41,42]. Thus, in the present study, the MSST method is applied to obtain the shock Hugoniot data in SC and nanocrystalline (NC) iron.

In the literature $[3,9,12,13,15,16]$, there are relatively few studies on the comparison between the shock Hugoniots, and EOS of SC and polycrystalline iron. The EOS, shock Hugoniot, and $T_{m}$ are significant physical properties that must be examined to study the behavior of metal materials under high-pressure conditions. There are two issues involved in the relation between shock Hugoniots and EOS: one regards obtaining the EOS based on the shock Hugoniots, and the other concerns the determination of the shock Hugoniots based on the EOS. In addition, the Grüneisen parameter and melting temperature can be calculated from the shock Hugoniot data. The investigation path of this work firstly utilizes MD simulation to obtain the shock Hugoniots of SC and NC iron; thus, the Hugoniot curve can be obtained. Therefore, based on the Hugoniot data, $\gamma$ and $T_{m}$ can be determined. Finally, the EOS of SC and NC iron can be presented.

\section{Methodology}

\subsection{Hugoniot Pressure $\left(P_{H}\right)$ and Internal Energy $\left(E_{H}\right)$}

The Hugoniot relation can be expressed by three conservation equations: the mass equation, the momentum equation, and the energy equation:

$$
\begin{gathered}
\rho\left(u_{s}-u_{p}\right)=\rho_{0} u_{s} \\
P_{H}-P_{0}=\rho_{0} u_{s} u_{p} \\
E_{H}-E_{0}=\frac{1}{2}\left(P_{H}+P_{0}\right)\left(V_{0}-V\right)
\end{gathered}
$$

where $\rho, u_{s}, u_{p}, P_{H}, E_{H}$, and $V(=1 / \rho)$ are the density, shockwave velocity, particle velocity, pressure, and internal energy per unit mass and specific volume of the shocked material, respectively. The shock wave velocity in the MD simulation was set to $6-11 \mathrm{~km} / \mathrm{s}$, and the particle velocity under different loading conditions was obtained by varying the loading velocity. The subscript " 0 " denotes the qualities in the initial state without shock, and " $H$ " represents the quantities in the Hugoniot state. For example, $P_{H}$ represents the pressure in the Hugoniot state.

It has been found that under a wide range of pressure, the relationship between the shockwave velocity $u_{s}$ and the particle velocity $u_{p}$ is approximately linear [11]:

$$
u_{s}=C_{0}+\lambda u_{p}
$$

where $C_{0}$ and $\lambda$ are the volume sound speed at zero pressure and the fitting parameter, respectively. From MD simulation results, $C_{0}$ and $\lambda$ of SC and NC iron can be determined. Once $C_{0}$ and $\lambda$ is determined, it can be used to calculate the Hugoniot curve $P_{H}(V)$ and the internal energy $E_{H}(V)$.

According to Equations (1)-(4), when $P_{0}=0$, the Hugoniot curve $P_{H}(V)$ and the internal energy $E_{H}(V)$ can be derived:

$$
\begin{gathered}
P_{H}(V)=\frac{\rho_{0} C_{0}^{2}\left(1-V / V_{0}\right)}{\left[1-\lambda\left(1-V / V_{0}\right)\right]^{2}} \\
E_{H}=E_{0}+\frac{1}{2} P_{H}\left(V_{0}-V\right)=\int_{0}^{T_{0}} c_{v} d T+\frac{1}{2} P_{H}\left(V_{0}-V\right)
\end{gathered}
$$

where $c_{v}$ and $T_{0}$ are the specific heat at constant volume and the initial temperature, respectively. If the $E_{H}$ is much larger than $E_{0}, E_{0}$ can usually be ignored. 


\subsection{Cold Pressure $\left(P_{c}\right)$ and Cold Energy $\left(E_{c}\right)$}

From the Hugoniot relations at room temperature (usually $300 \mathrm{~K}$ ), $V_{0 K}, \rho_{0 K}, C_{0}^{\prime}$, and $\lambda^{\prime}$ can be obtained as follows [43]:

$$
\begin{gathered}
V_{0 K}=V_{0}\left(1-\alpha_{v} T_{0}\right) \\
\rho_{0 K}=\frac{\rho_{0}}{1-\alpha_{v} T} \\
C_{0}^{\prime 2}=C_{0}^{2} \frac{(1-F)^{2}}{(1-F \lambda)^{3}}\left[1-F \cdot \gamma\left(V_{0}\right)+F \lambda\right] \\
\lambda^{\prime}=\lambda\left(\frac{C_{0}}{C_{0}^{\prime}}\right)^{2} \frac{(1-F)^{3}}{(1-F \lambda)^{4}}\left[\left(1+\frac{F \lambda}{2}\right)\left(1-\frac{F \gamma\left(V_{0}\right)}{2}\right)-\frac{F \gamma^{2}\left(V_{0}\right)}{4 \lambda}(1-F \lambda)\right] \\
F=\int_{0}^{300} \alpha_{v}(T) d T
\end{gathered}
$$

where $\gamma\left(V_{0}\right)$ is the Grüneisen coefficient at room temperature (usually $300 \mathrm{~K}$ ); $\alpha_{v}(T)$ is the volume expansion coefficient, which is approximately constant from $0 \mathrm{~K}$ to $300 \mathrm{~K}$; and $C_{0}^{\prime}$, and $\lambda^{\prime}$ are parameters of $u_{s}$ and $u_{p}$ relations at $0 \mathrm{~K}$.

The Born-Mayer [44] and Morse [45] potentials are usually applied to describe the atomic forces at $0 \mathrm{~K}$; thus, the cold pressure $P_{c}$ and cold energy $E_{c}$ can be expressed as follows:

$$
\begin{gathered}
P_{c-B M}=Q \delta^{2 / 3}\left\{\exp \left[q\left(1-\delta^{-1 / 3}\right)\right]-\delta^{2 / 3}\right\} \\
E_{c-B M}=\frac{3 Q}{\rho_{0 K}}\left\{\frac{1}{q} \cdot \exp \left[q\left(1-\delta^{-1 / 3}\right)\right]-\delta^{1 / 3}-\left(\frac{1}{q}-1\right)\right\} \\
P_{c-M}=A \delta^{2 / 3}\left[e^{2 B\left(1-\delta^{-1 / 3}\right)}-e^{B\left(1-\delta^{-1 / 3}\right)}\right] \\
E_{c-M}=\frac{3 A}{2 \rho_{0 K} B}\left[e^{B\left(1-\delta^{-1 / 3}\right)}-1\right]^{2}
\end{gathered}
$$

where $Q, q, A$, and $B$ are material constants; $\delta=V_{0 K} / V$; and $\rho_{0 K}$ is the material density at 0 .

At $P=0$ and $T=0$, we can assume that the first-order and second-order derivatives of $P_{H}$, isentropic $P_{s}$, and $P_{c}$ are almost equal $[5,46]$. Thus, we have:

$$
\begin{gathered}
\left(\frac{\partial P_{H}}{\partial V}\right)_{V_{0 K}}=\left(\frac{\partial P_{s}}{\partial V}\right)_{V_{0 K}} \approx\left(\frac{\partial P_{c}}{\partial V}\right)_{V_{0 K}} \\
\left(\frac{\partial^{2} P_{H}}{\partial V^{2}}\right)_{V_{0 K}}=\left(\frac{\partial^{2} P_{s}}{\partial V^{2}}\right)_{V_{0 K}} \approx\left(\frac{\partial^{2} P_{c}}{\partial V^{2}}\right)_{V_{0 K}}
\end{gathered}
$$

With the determined $C_{0}$ and $\lambda$ from MD simulations, and according to Equations (5) and (10)-(15), $Q, q, A$, and $B$ can be calculated as follows:

$$
\begin{gathered}
Q=\frac{3 C_{0}^{\prime 2} \rho_{0 K}}{q-2} \\
q=6 \lambda^{\prime}-3+\sqrt{3\left(12 \lambda^{\prime 2}-20 \lambda^{\prime}+9\right)} \\
A=\frac{3 \rho_{0 K} C_{0}^{\prime 2}}{4 \lambda^{\prime}-2} \\
B=4 \lambda^{\prime}-2
\end{gathered}
$$




\subsection{Grüneisen Coefficient $\gamma$}

The Grüneisen coefficient $\gamma$ shows a dependence of volume and can be expressed as follows [30]:

$$
\gamma(V)=\frac{t-2}{3}-\frac{V}{2} \frac{d^{2}\left[P_{c}(V) V^{2 t / 3}\right] / d V^{2}}{d\left[P_{c}(V) V^{2 t / 3}\right] / d V}
$$

where $t$ is used to represent three models for characterizing $\gamma$. When $t=0$, Equation (23) represents the Slater model [27] $\gamma_{s}(V) ; t=1$ represents the Dugdale-MacDonald model [28] $\gamma_{D M}(V)$; and $t=2$ represents the free-volume model [29] $\gamma_{f}(V)$.

At $P=0$ and $V=V_{0}$, Equation (22) approximately satisfies the isentropic $P_{S}(V)$, and, consequently, Equation (22) can be expressed as follows:

$$
\gamma_{s}\left(V_{0}\right)=-\frac{t+2}{3}-\frac{V_{0}}{2} \frac{P_{s}^{\prime \prime}\left(V_{0}\right)}{P_{s}^{\prime}\left(V_{0}\right)}
$$

When $t$ is equal to 0,1 , and 2 :

$$
\begin{aligned}
& \gamma_{s}\left(V_{0}\right)_{t=0}=-\frac{2}{3}-\frac{V_{0}}{2} \frac{P_{s}^{\prime \prime}\left(V_{0}\right)}{P_{s}^{\prime}\left(V_{0}\right)} \\
& \gamma_{D M}\left(V_{0}\right)_{t=1}=-1-\frac{V_{0}}{2} \frac{P_{s}^{\prime \prime}\left(V_{0}\right)}{P_{s}^{\prime}\left(V_{0}\right)} \\
& \gamma_{f}\left(V_{0}\right)_{t=2}=-\frac{4}{3}-\frac{V_{0}}{2} \frac{P_{s}^{\prime \prime}\left(V_{0}\right)}{P_{s}^{\prime}\left(V_{0}\right)}
\end{aligned}
$$

When $P=0$ and $V=V_{0}$, the first-order and second-order derivatives of $P_{S}(V)$ and $P_{H}(V)$ are identical. In addition, $P_{H}^{\prime}\left(V_{0}\right)$ and $P_{H}^{\prime \prime}\left(V_{0}\right)$ can be derived from $P_{H}$ obtained via MD simulations or experiments; therefore, we have:

$$
\begin{gathered}
P_{S}^{\prime}\left(V_{0}\right)=P_{H}^{\prime}\left(V_{0}\right)=-\left(\frac{C_{0}}{V_{0}}\right)^{2} \\
P_{S}^{\prime \prime}\left(V_{0}\right)=P_{H}^{\prime \prime}\left(V_{0}\right)=4\left(\frac{C_{0}^{2} \lambda}{V_{0}^{3}}\right) \\
\frac{P_{S}^{\prime \prime}\left(V_{0}\right)}{P_{S}^{\prime}\left(V_{0}\right)}=\frac{P_{H}^{\prime \prime}\left(V_{0}\right)}{P_{H}^{\prime}\left(V_{0}\right)}=-\frac{4 \lambda}{V_{0}}
\end{gathered}
$$

Substituting Equations (27)-(29) into Equation (23) yields

$$
\begin{gathered}
\gamma_{s}\left(V_{0}\right)=2 \lambda-\frac{2}{3} \\
\gamma_{D M}\left(V_{0}\right)=2 \lambda-1 \\
\gamma_{f}\left(V_{0}\right)=2 \lambda-\frac{4}{3} \\
\gamma_{s}\left(V_{0}\right)=\gamma_{D M}\left(V_{0}\right)+\frac{1}{3}=\gamma_{f}\left(V_{0}\right)+\frac{2}{3}
\end{gathered}
$$

As we have determined $\lambda$ from MD simulations, the value of Equations (30)-(32) can be obtained. From previous work, the volume dependence of $\gamma$ can be commonly expressed by the following simpler empirical formulas [5,47-49]:

$$
\gamma(V)=\gamma_{0} \frac{V}{V_{0}}
$$




$$
\begin{gathered}
\gamma(V)=\frac{2}{3}+\left(\gamma_{0}-\frac{2}{3}\right) \frac{V}{V_{0}} \\
\gamma(V)=\frac{2}{3}+\left(\gamma_{0}-\frac{2}{3}\right)\left(\frac{V}{V_{0}}\right)^{1.18} \\
\gamma(V)=0.67+\left(\gamma_{0}-0.67\right)\left(\frac{V}{V_{0}}\right)^{1.46} \\
\gamma(V)=\frac{1}{2}+0.86\left(\frac{V}{V_{0}}\right)^{1 / 3}+0.25\left(\frac{V}{V_{0}}\right)^{4.5}
\end{gathered}
$$

\subsection{Melting Temperature $\left(T_{m}\right)$}

In high-pressure physics, the melting temperature $T_{m}(V)$ is a significant material characteristic. The Lindemann melting criterion is given by [50]:

$$
\frac{d \ln T_{m}(V)}{d \ln V}=-\frac{2}{3}+2 \gamma(V)
$$

According to Equation (39), Equations (40)-(44) are deduced as:

$$
\begin{gathered}
T_{m}(V)=T_{m 0}\left(\frac{V}{V_{0}}\right)^{2 / 3} \exp \left[2 \gamma_{0}\left(1-\frac{V}{V_{0}}\right)\right] \\
T_{m}(V)=T_{m 0}\left(\frac{V}{V_{0}}\right)^{-2 / 3} \exp \left[2\left(\gamma_{0}-2 / 3\right)\left(1-\frac{V}{V_{0}}\right)\right] \\
T_{m}(V)=T_{m 0}\left(\frac{V}{V_{0}}\right)^{-2 / 3} \exp \left\{\frac{2\left(\gamma_{0}-2 / 3\right)}{1.18}\left[1-\left(\frac{V}{V_{0}}\right)^{1.18}\right]\right\} \\
T_{m}(V)=T_{m 0}\left(\frac{V}{V_{0}}\right)^{-0.67} \exp \left\{\frac{2\left(\gamma_{0}-0.9\right)}{1.46}\left[1-\left(\frac{V}{V_{0}}\right)^{1.46}\right]\right\} \\
T_{m}(V)=T_{m 0}\left(\frac{V}{V_{0}}\right)^{-1 / 3} \exp \left\{6 \times 0.86 \times\left[1-\left(\frac{V}{V_{0}}\right)^{1 / 3}\right]+\frac{2 \times 0.25}{4.5}\left[1-\left(\frac{V}{V_{0}}\right)^{4.5}\right]\right\}
\end{gathered}
$$

where $\gamma_{0}$ is determined from Equations (30)-(32), $T_{m 0}$ is the melting temperature at $0 \mathrm{GPa}$, and is $1811 \mathrm{~K}$ for iron [51].

\subsection{Mie-Grüneisen Equation of State}

The Mie-Grüneisen EOS is usually applied to describe the behavior of metal materials under extreme shock compression, and the form of Mie-Grüneisen EOS can be expressed as follows [24]:

$$
\begin{gathered}
P-P_{c}=\frac{\gamma(V)}{V}\left(E-E_{c}\right) \\
P-P_{H}=\frac{\gamma(V)}{V}\left(E-E_{H}\right)
\end{gathered}
$$

From the calculation results of $P_{H}, E_{H}, P_{c}, E_{c}$, and $\gamma(V)$, the Mie-Grüneisen EOS could be obtained.

\section{MD Simulation}

The embedded-atom method (EAM) potential presented by Mendelev et al. [52] was used to conduct the MD simulation in this study. Three simulation models were established to investigate the dynamic characteristics of SC and NC iron under intensive shock conditions. The established models were based on bcc iron, and the shockwave direction is [001] in SC iron. Figure 1 shows the three simulation models. The NC models 
were built on the Voronoi algorithm in Atomsk [53]. The periodical boundary conditions were set in all directions for both SC and NC iron. To balance the calculation time and model size, the simulation system of SC iron was about $8.4 \times 8.4 \times 8.4 \mathrm{~nm}^{3}$ with 54,000 atoms. The model sizes of NC iron were about $10.9 \times 10.9 \times 10.9 \mathrm{~nm}^{3}$ with 111,195 atoms and $54.7 \times 54.7 \times 54.7 \mathrm{~nm}^{3}$ with 8,268,098 atoms. There were two grain sizes (GSs): $5 \mathrm{~nm}$ and $25 \mathrm{~nm}$, which were chosen to study the GS effect in the shocking behavior.
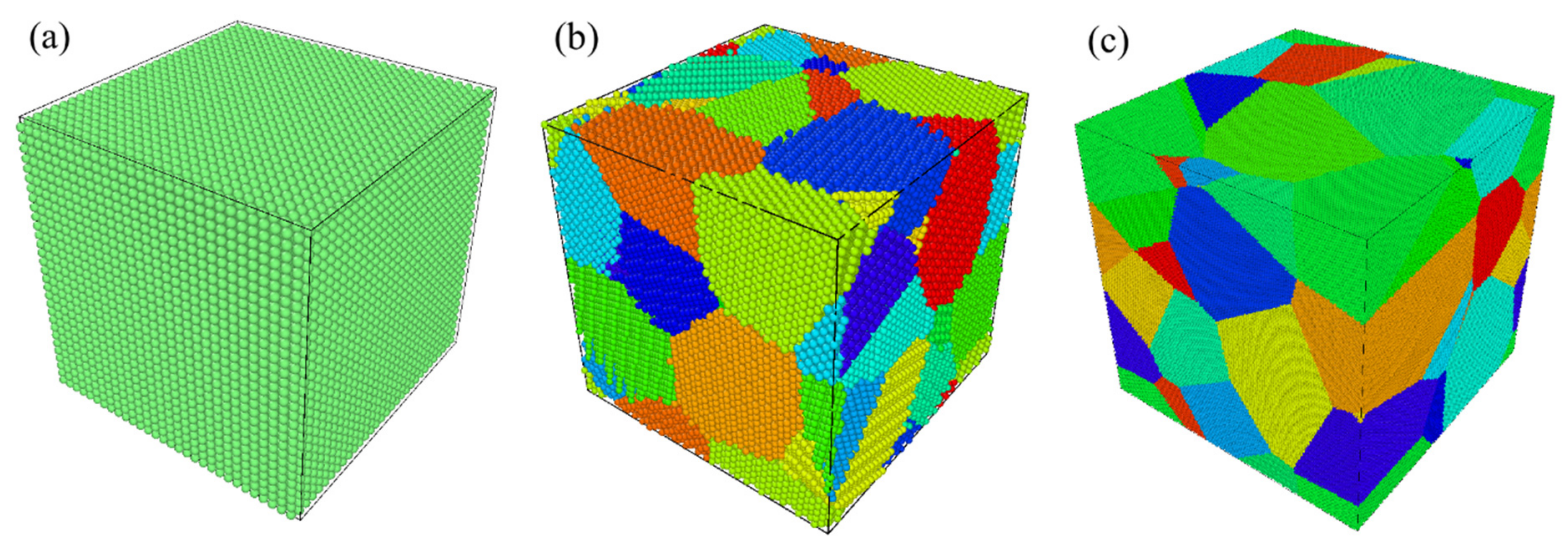

Figure 1. Configurations of (a) SC iron and (b) NC iron with GS of $5 \mathrm{~nm}$ and (c) NC iron with GS of $25 \mathrm{~nm}$. Separate grains are distinguished by different colors.

First, the simulation system was equilibrated at $300 \mathrm{~K}$ for $10 \mathrm{ps}$ in the NPT ensemble to ensure that the system reached a steady state in order to eliminate the residual stress. Then, the shockwave was examined using the MSST method under velocities of 6-11 km/s. Moreover, the computation time should be long enough to guarantee that the shockwave in the system reaches a steady state after the shock. Thus, the statistical results, such as the particle velocity, pressure, and temperature, could be obtained from the MD results.

\section{Results and Discussion}

\subsection{Shock Hugoniot Pressure and Internal Energy}

According to the experimental Hugoniot data from Brown et al. [11], the shock velocity $u_{s}$ ranged from 5.3 to $11.5 \mathrm{~km} / \mathrm{s}$ at about $40-440 \mathrm{GPa}$. To ensure the stability of the MD simulation and evaluate the model validity, the velocity of $u_{s}$ was set in the range of 6 to $11 \mathrm{~km} / \mathrm{s}$ in this study. Figure 2 shows the relationship between $u_{s}$ and $u_{p}$ for SC iron obtained via the MSST method; there is a good agreement between the MD simulation results and experimental data. Moreover, the linear relationship between $u_{s}$ and $u_{p}$ is in excellent accordance with the data in Figure 2. The linear relationship is also consistent with the study by Prieto and Renero [54], which indicates that the relationship between $u_{s}$ and $u_{p}$ of iron below 5 megabars can be described by a linear relationship. For SC iron, the relationship between $u_{s}$ and $u_{p}$ is given as $u_{s}=4.071+1.538 u_{p}$. The relationship between $u_{s}$ and $u_{p}$ from the experimental data is expressed as $u_{s}=3.935+1.578 u_{p}$. The relative errors of $C_{0}$ and $\lambda$ obtained from the MSST method and experiment were 3.3\% and 2.6\%, respectively, which proves the accuracy of the MD simulation.

To study the GS effect on the $u_{s}-u_{p}$ relationship for NC iron, MD simulation was conducted at the same shock velocity as that of SC iron. The results are shown in Figure 3. The results of NC iron with different GS values are close. These discrete data are characterized by $u_{s}=4.011+1.552 u_{p}$ and $u_{s}=4.01+1.553 u_{p}$ for GS $5 \mathrm{~nm}$ and GS $25 \mathrm{~nm}$, respectively. The relative error of $C_{0}$ and $\lambda$ between GS $5 \mathrm{~nm}$ and GS $25 \mathrm{~nm}$ was less than $0.1 \%$, and compared with the SC iron data, $C_{0}$ and $\lambda$ were approximately equal to an error of $<1.5 \%$. The results indicate that the GS effect had little influence on the linear relationship between $u_{s}$ and $u_{p}$ for SC and NC iron, and it could be neglected in the case of NC iron. 


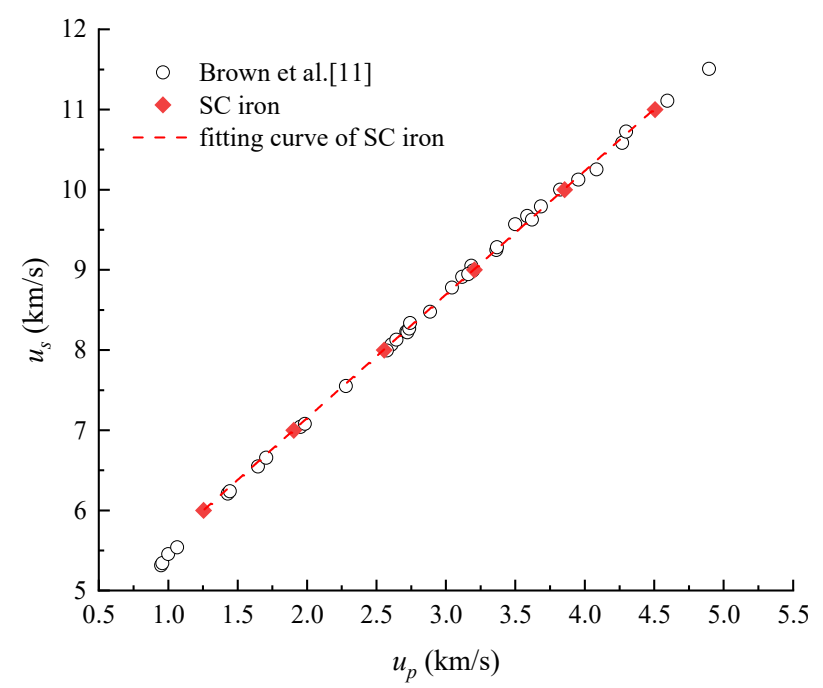

Figure 2. The relation between $u_{s}$ and $u_{p}$ for SC at $300 \mathrm{~K}$ (the linear fitting curve is applied to the MD results; experimental data are shown for comparison).
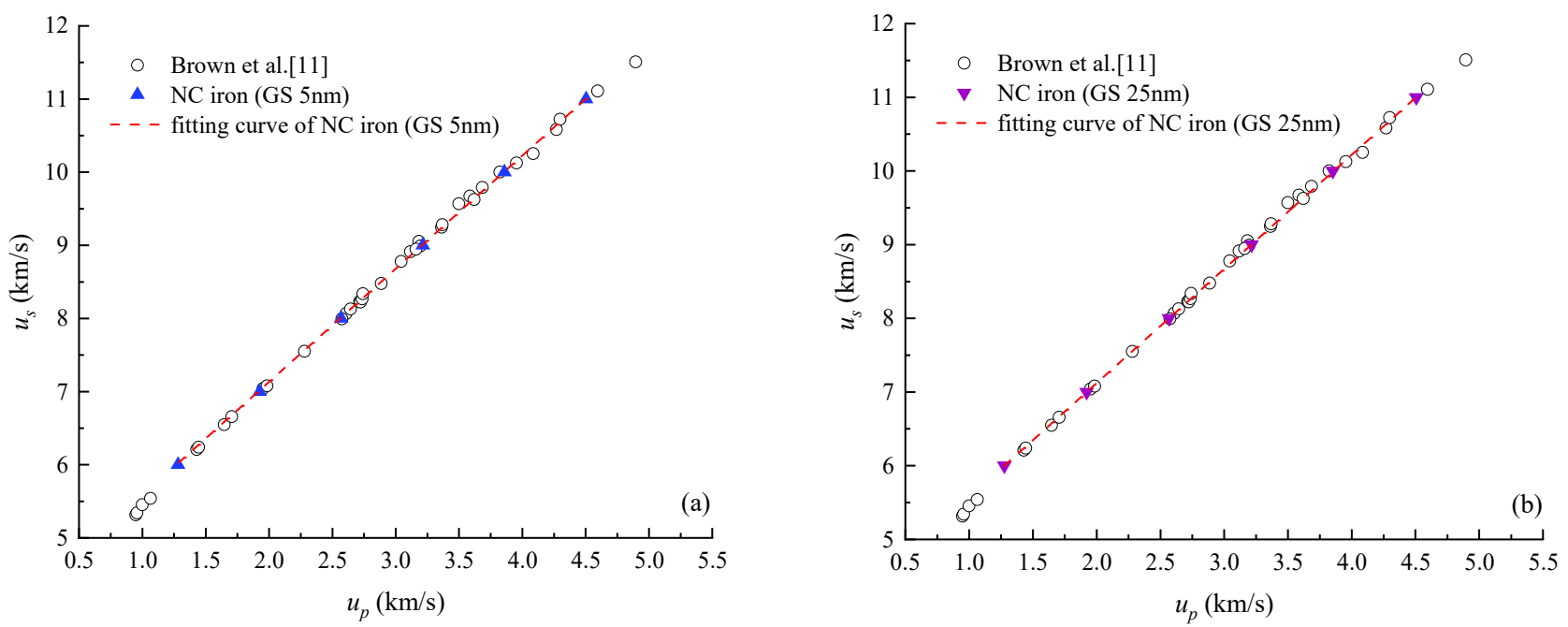

Figure 3. The relationship between $u_{s}$ and $u_{p}$ for NC iron at $300 \mathrm{~K}$ : (a) NC iron with GS $5 \mathrm{~nm}$ (b) NC iron with GS $25 \mathrm{~nm}$ (the linear fitting curve is applied to the MD results; experimental data are shown for comparison).

The shock Hugoniot data obtained from MD simulations of SC and NC iron are listed in Table 1. As can be seen in Table 1, the particle velocity is high in NC iron below a shock velocity of $8.0 \mathrm{~km} / \mathrm{s}$, with a maximum difference of $2.5 \%$ between SC and NC iron. However, as the shock speed continues to grow, the difference in particle velocity becomes very small. At $11 \mathrm{~km} / \mathrm{s}$, the maximum shock pressure in SC and NC iron is 387.98-389.62 GPa. From [11], it can be found that the shock pressure is $361.8 \mathrm{GPa}$ at a shock velocity of $10.7 \mathrm{~km} / \mathrm{s}$ and $400.7 \mathrm{GPa}$ at a shock velocity of $11.11 \mathrm{~km} / \mathrm{s}$. The MD results are within the range of the experimental data, which shows the accuracy of the simulation.

A detailed comparison of MD simulation results and experimental data is presented in Table 2. From Table 2, it can be observed that the volume sound speed $C_{0}$ in SC iron is higher than that in NC iron; however, the fitting parameter $\lambda$ in SC iron is lower than that in $\mathrm{NC}$ iron, and the same characteristics are also observed in SC and NC Al [5]. The maximum relative error of $C_{0}$ between the experiment and SC iron is $3.34 \%$, and the maximum relative error of NC iron is $1.89 \%$. The difference of $C_{0}$ between SC and NC iron is $\sim 1.5 \%$, which is less than the difference with experiment data. The same characteristics in $\lambda$ can be observed; the maximum relative error between the experiment and SC iron is $2.60 \%$, and the maximum relative error of NC iron is $1.67 \%$. The difference of $\lambda$ between SC and NC 
iron is $\sim 0.96 \%$. The results indicate that a shock response difference between SC and NC iron does exist, but the difference is not very significant.

Table 1. Hugoniot data for SC and NC iron determined by MD simulations at $300 \mathrm{~K}$. An initial density of $7.85 \mathrm{~g} / \mathrm{cm}^{3}$ is used.

\begin{tabular}{|c|c|c|c|c|}
\hline Material & $u_{p}(\mathrm{~km} / \mathrm{s})$ & $u_{s}(\mathrm{~km} / \mathrm{s})$ & $P(\mathrm{GPa})$ & $\rho\left(\mathrm{g} / \mathrm{cm}^{3}\right)$ \\
\hline \multirow{6}{*}{ SC iron } & 1.25 & 6.00 & 60.84 & 9.92 \\
\hline & 1.90 & 7.00 & 102.92 & 10.78 \\
\hline & 2.55 & 8.00 & 158.50 & 11.53 \\
\hline & 3.20 & 9.00 & 224.72 & 12.19 \\
\hline & 3.85 & 10.00 & 301.71 & 12.77 \\
\hline & 4.50 & 11.00 & 389.62 & 13.29 \\
\hline \multirow{6}{*}{ NC iron (GS $5 \mathrm{~nm}$ ) } & 1.27 & 6.00 & 62.36 & 9.98 \\
\hline & 1.92 & 7.00 & 105.82 & 10.82 \\
\hline & 2.57 & 8.00 & 161.41 & 11.56 \\
\hline & 3.21 & 9.00 & 227.10 & 12.21 \\
\hline & 3.85 & 10.00 & 302.92 & 12.78 \\
\hline & 4.50 & 11.00 & 388.85 & 13.29 \\
\hline \multirow{6}{*}{ NC iron (GS $25 \mathrm{~nm}$ ) } & 1.28 & 6.00 & 62.34 & 9.98 \\
\hline & 1.92 & 7.00 & 105.74 & 10.82 \\
\hline & 2.56 & 8.00 & 161.22 & 11.56 \\
\hline & 3.21 & 9.00 & 226.75 & 12.20 \\
\hline & 3.85 & 10.00 & 302.34 & 12.77 \\
\hline & 4.50 & 11.00 & 387.98 & 13.28 \\
\hline
\end{tabular}

Table 2. $C_{0}$ and $\lambda$ obtained from the linear relationship between $u_{s}$ and $u_{p}$ for SC and NC iron at $300 \mathrm{~K}$. The initial density is $7.85 \mathrm{~g} / \mathrm{cm}^{3}$ in both the experiment and MD simulation.

\begin{tabular}{|c|c|c|c|c|c|c|c|c|c|c|}
\hline \multirow{3}{*}{ Material } & \multirow{3}{*}{$\begin{array}{l}\text { Shock } \\
\text { Velocity }\end{array}$} & \multirow{3}{*}{$\begin{array}{c}C_{0} \\
(\mathrm{~km} / \mathrm{s})\end{array}$} & \multirow{3}{*}{$\lambda$} & \multicolumn{6}{|c|}{ Relative Error } & \multirow{3}{*}{ Reference } \\
\hline & & & & \multicolumn{2}{|c|}{ SC } & \multicolumn{2}{|c|}{ NC (GS $5 \mathrm{~nm})$} & \multicolumn{2}{|c|}{ NC (GS 25 nm) } & \\
\hline & & & & $C_{0}$ & $\lambda$ & $C_{0}$ & $\lambda$ & $C_{0}$ & $\lambda$ & \\
\hline Commercial iron & $5.3-11.5 \mathrm{~km} / \mathrm{s}$ & 3.935 & 1.578 & $3.34 \%$ & $2.60 \%$ & $1.89 \%$ & $1.67 \%$ & $1.87 \%$ & $1.61 \%$ & [11] \\
\hline SC & $6-11 \mathrm{~km} / \mathrm{s}$ & 4.071 & 1.538 & - & - & $1.49 \%$ & $0.90 \%$ & $1.50 \%$ & $0.96 \%$ & This work \\
\hline $\mathrm{NC}(\mathrm{GS} 5 \mathrm{~nm})$ & $6-11 \mathrm{~km} / \mathrm{s}$ & 4.011 & 1.552 & $1.49 \%$ & $0.90 \%$ & - & - & $0.02 \%$ & $0.06 \%$ & This work \\
\hline $\mathrm{NC}$ (GS $25 \mathrm{~nm})$ & $6-11 \mathrm{~km} / \mathrm{s}$ & 4.010 & 1.553 & $1.50 \%$ & $0.96 \%$ & $0.02 \%$ & $0.06 \%$ & - & - & This work \\
\hline
\end{tabular}

Based on the linear relationship obtained for SC and NC iron, the Hugoniot curve could be obtained using Equations (5) and (6). Figure 4 shows the Hugoniot curves of SC and NC iron. The black circle represents the results by Brown et al. [11], and the colored curves represent this work for SC and NC iron. Figure 4a shows that for $V / V_{0}>0.6$, the curves obtained through the MSST method agree well with the results of Brown et al. [11]; when $V / V_{0}<0.6$, the curves are approximately equal to the experiment results, and the deviation is less than $3 \%$. Considering the insignificant error of $C_{0}$ and $\lambda$ for SC and NC iron, there is no noticeable difference between the three curves in Figure 4a. As shown in Figure $4 \mathrm{~b}$, the internal energies obtained in the MD simulation and experiment were almost the same; when $V_{0} / V=1.7$, the internal energy of SC iron was smaller than that of $\mathrm{NC}$ iron, and the deviation at $V_{0} / V=1.7$ was $0.5 \%$. The results not only prove the reliability of the linear relationship between $u_{s}$ and $u_{p}$ but also show the EAM potential for iron in the MD simulation. In addition, the results signify that the MD results are appropriate for describing the cold pressure, the cold energy, the melting temperature, and the Mie-Grüneisen EOS. 

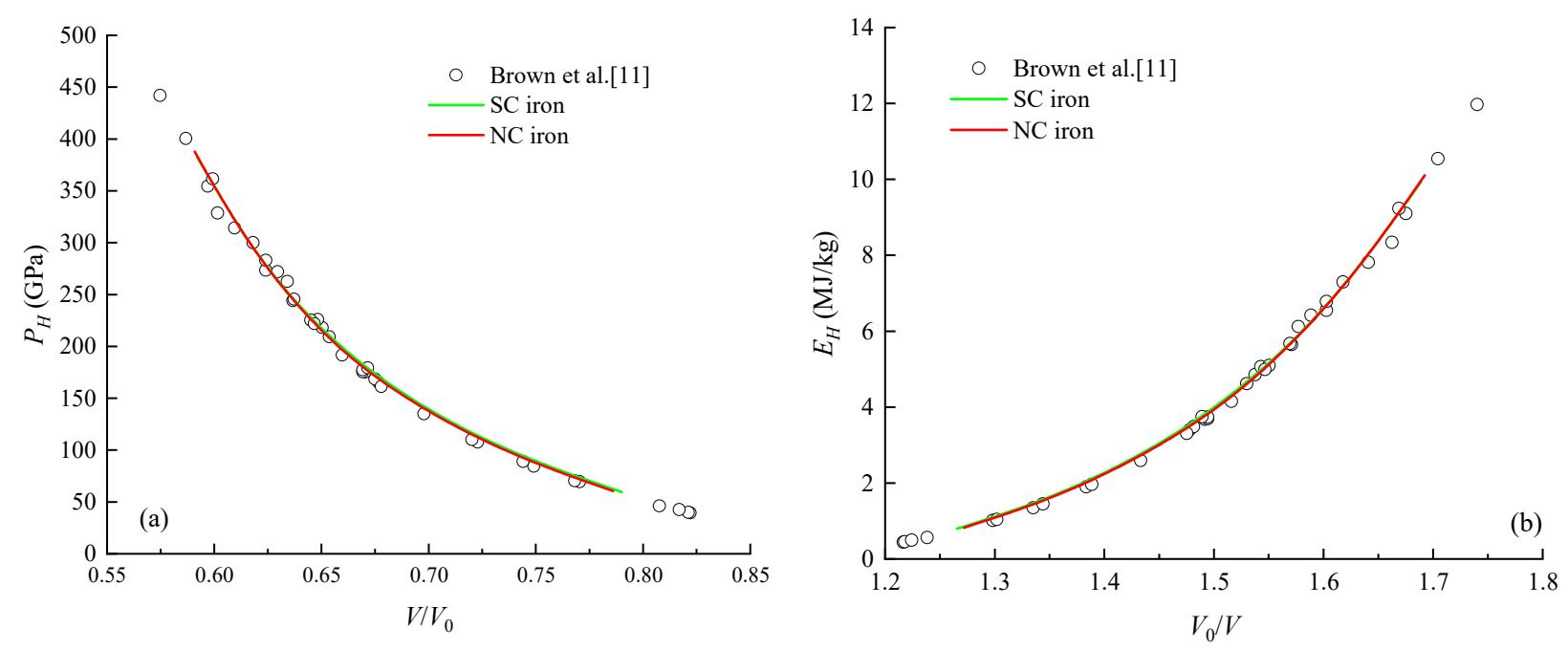

Figure 4. The Hugoniot curves of SC and NC iron: (a) $P_{H}$ curve; (b) $E_{H}$ curve.

\subsection{Cold Pressure and Cold Energy}

Based on the $C_{0}$ and $\lambda$ obtained from the Hugoniot data, the material constants for calculating cold pressure and cold energy in Equations (18)-(21) could be obtained. The values are presented in Table 3.

Table 3. Material constants for calculating cold pressure and cold energy.

\begin{tabular}{cccccccc}
\hline $\begin{array}{c}\text { Material } \\
\text { Constants }\end{array}$ & $\boldsymbol{V}_{\mathbf{0}}\left(\mathbf{g} / \mathbf{c m}^{\mathbf{3}}\right)$ & $\left.\boldsymbol{C}_{0}^{\prime} \mathbf{( k m} / \mathbf{s}\right)$ & $\lambda^{\prime}$ & $\boldsymbol{Q}(\mathrm{GPa})$ & $\boldsymbol{q}$ & $\boldsymbol{A}(\mathrm{GPa})$ & $\boldsymbol{B}$ \\
\hline Experiment & 0.127 & 4.010 & 1.581 & 41.245 & 11.192 & 87.662 & 4.325 \\
SC iron & 0.127 & 4.094 & 1.540 & 46.473 & 10.716 & 97.319 & 4.162 \\
NC iron & 0.127 & 4.093 & 1.541 & 44.238 & 10.894 & 93.170 & 4.223 \\
\hline
\end{tabular}

Figures 5 and 6 compare the cold pressure $P_{c}$ and cold energy $E_{c}$ between SC and NC iron, with the experimental data as a reference. The solid and dashed curves represent $P_{c}$ and $E_{c}$ obtained using the Born-Mayer and Morse potentials, respectively. The results from the Born-Mayer and Morse potentials presented the same increasing trend: $P_{c}$ and $E_{c}$ increased with volume compression $\left(V_{0 \mathrm{~K}} / V\right)$, which presented a sharp increment at higher compression.

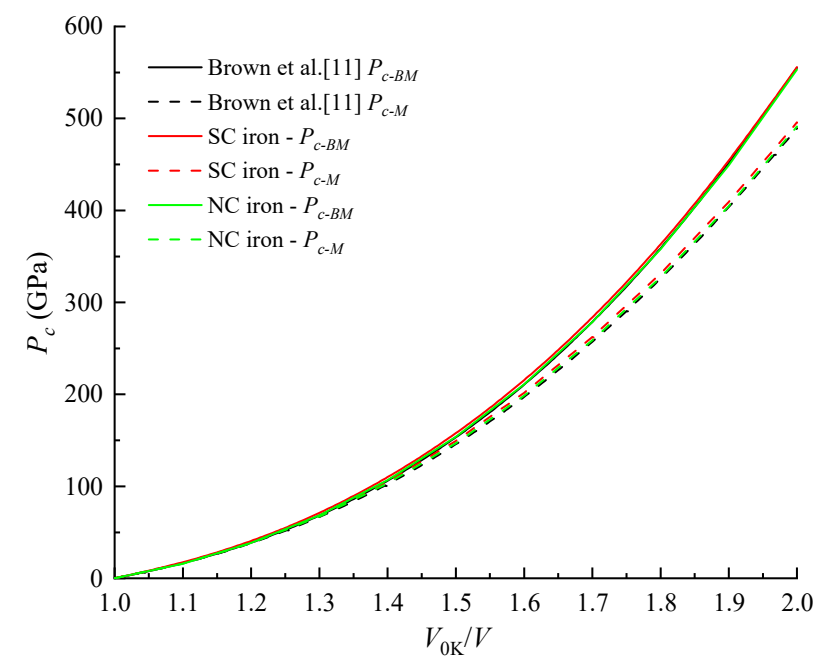

Figure 5. The cold pressures of SC and NC iron. 


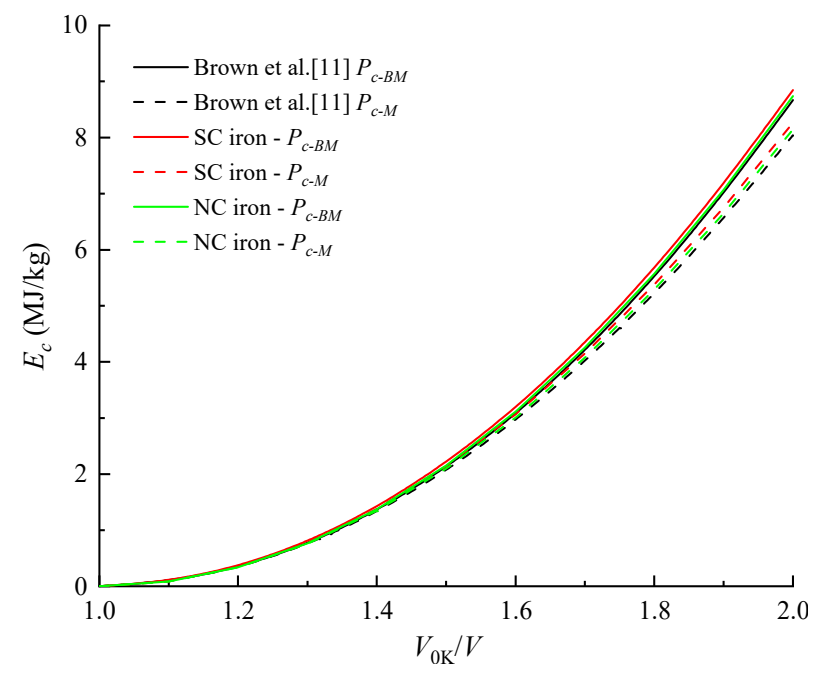

Figure 6. The cold energy of SC and NC iron.

At $V_{0 \mathrm{~K}} / V$ less than 1.3, the curves of $P_{c}$ agree well with each other. The result shows that the cold energies described by the Born-Mayer and Morse potentials are basically the same in the low-compression region. When the $V_{0 \mathrm{~K}} / V$ is above 1.3 , the $P_{c}$ curves gradually deviate from each other, and the $P_{c}$ curves obtained by the Born-Mayer potential gradually become larger. The relative error of $P_{c}$ was about $10 \%$ when $V_{0 \mathrm{~K}} / V$ was 2.0. Irrespective of the potential function used, the cold pressure curve of SC iron was above that of the NC iron. This indicates that the cold pressure of SC iron was higher than that of NC iron, although the difference was quite small.

Under $V_{0 K} / V$ of less than 1.4, the cold energy curves agree well with each other, as the cold pressures obtained by the Born-Mayer and Morse potentials in the low-compression region are approximately equal. Under $V_{0 K} / V$ of above 1.4 , the $E_{c}$ curves gradually deviate from each other. The cold energy obtained by the Born-Mayer potential is larger than that from the Morse potential, and the same trend was found for the cold pressure. The relative error of $E_{c}$ is about $7 \%$ when $V_{0 K} / V$ is 2.0. Furthermore, the cold energy in the SC iron is larger than that of the NC iron in both Born-Mayer and Morse potentials.

\subsection{Grüneisen Coefficient}

The material constant $\lambda$ in SC and NC iron was used to calculate the $\gamma_{0}$ of the Slater, Dugdale-Macdonald, and free-volume models through Equations (30)-(32). Subsequently, $\gamma_{0}(V)$ was substituted into Equations (34)-(38) to obtain $\gamma(V)$, and the experimental data were used for comparison. Figure 7 shows the results of $\gamma(V)$ from different empirical and theoretical models.

Equations (34)-(38) are commonly used empirical models for calculating $\gamma(V)$ at high pressure. From Figure 7, it can be seen that the Grüneisen coefficient $\gamma(V)$ first rapidly decreased and then slowly dropped as the $V_{0} / V$ increased. At high compression, the difference between $\mathrm{SC}$ and NC results became smaller; this indicates that the microstructure has little effect on $\gamma(V)$ under high pressure. Equation (38) is suitable for low-compression data, that is, $V_{0} / V$ up to 1.5 , and is free with $\lambda$; thus, different models do not affect their results. With Equation (38) as a reference, when $V_{0} / V=1.0$, the initial value of $\gamma_{0}$ is $\gamma_{f}\left(V_{0}\right)<\gamma_{D M}\left(V_{0}\right)<\gamma_{S}\left(V_{0}\right)$. For all three models, the $\gamma(V)$ obtained by the experimental data (solid curve) is at the top followed by that for the NC iron (dash curve), and that for the SC iron (dot curve) is at the bottom due to the difference between the $\lambda$ values. Compared with the results from Equations (35)-(38), the results from Equation (34) (black curves) show a more significant downward trend with the increase in $V_{0} / V$. This shows that the $\gamma(V)$ calculated using Equation (34) has the maximum change rate with the increase in $V_{0} / V$. The results from Equation (35)-(38) present a similar trend in predicting $\gamma(V)$. 

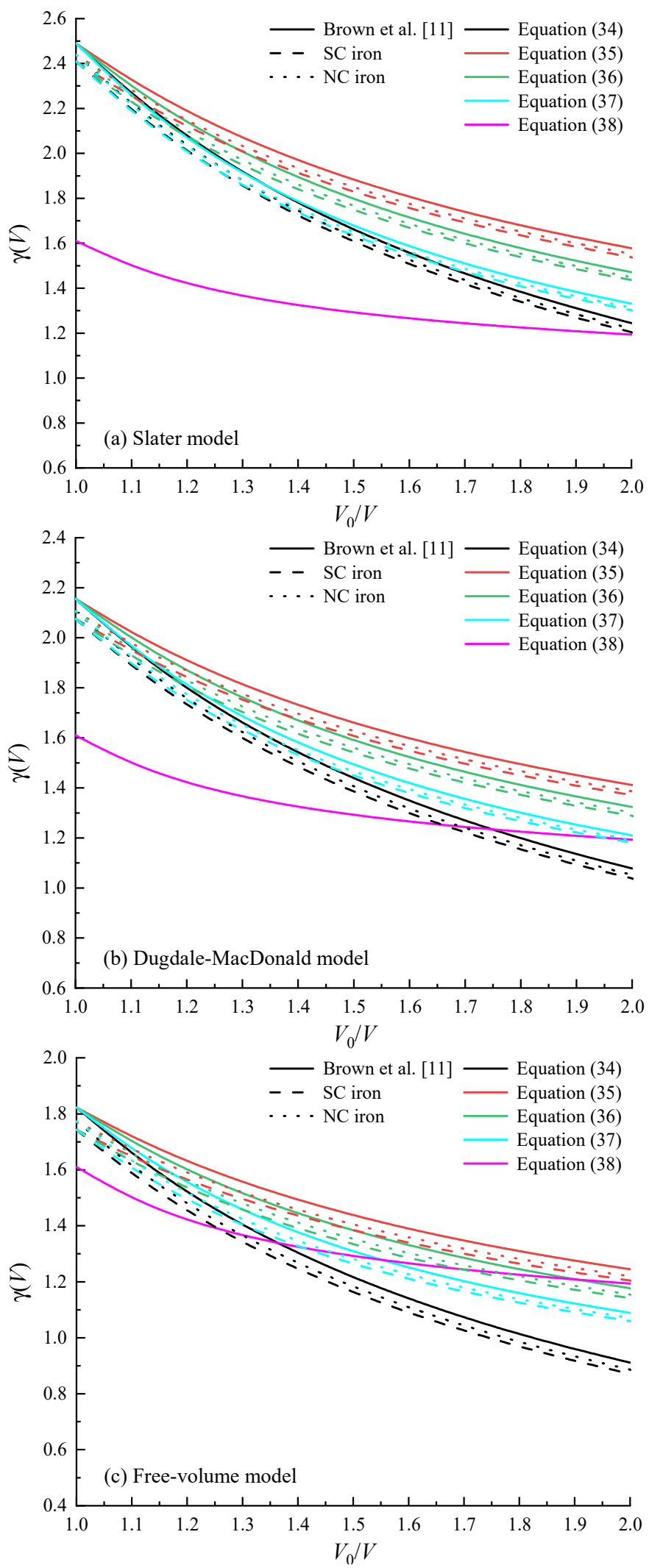

Figure 7. The relationship between the Grüneisen coefficient $\gamma(V)$ and $V_{0} / V$. (a) $\gamma(V)$ calculated from the Slate model, (b) $\gamma(V)$ calculated from the Dugdale-MacDonald model, (c) $\gamma(V)$ calculated from the Free-volume model. 
Equation (38) (magenta curve) is free of $\lambda$, and it has been proven to agree well with the experimental data under $V_{0} / V$ up to 1.5 [49]. The $\gamma(V)$ obtained using the free-volume model has the smallest difference under $V_{0} / V$ up to 1.5 . When $V_{0} / V$ is 1.5 , the $\gamma(V)$ obtained by Equation (37) (cyan curve) intersects with the magenta curve. This indicates that Equation (27) is applicable to characterize the $\gamma(V)$ of iron at higher compression compared with other expressions. This has also been proved by Jacobs and SchmidFetzer [48]. In Figure 7c, the green curve is closer to the cyan curve than other curves. The result shows that Equation (36) (green curve) is more suitable than other expressions for describing $\gamma(V)$. The relative error of the green curve is $7.8 \%$ at $V_{0} / V=2.0$.

\subsection{Melting Temperature}

Based on the Grüneisen coefficient and the Lindemann law, the melting temperature $T_{m}$ can be obtained. In this study, $\gamma_{0}$ is calculated via the free-volume model with Equation (26), and $T_{m}$ is predicted by Equations (40)-(44). The relationship between $T_{m}$ and pressure is illustrated in Figure 8, together with experimental data $[35,55-63]$ and theoretical data [64-68].

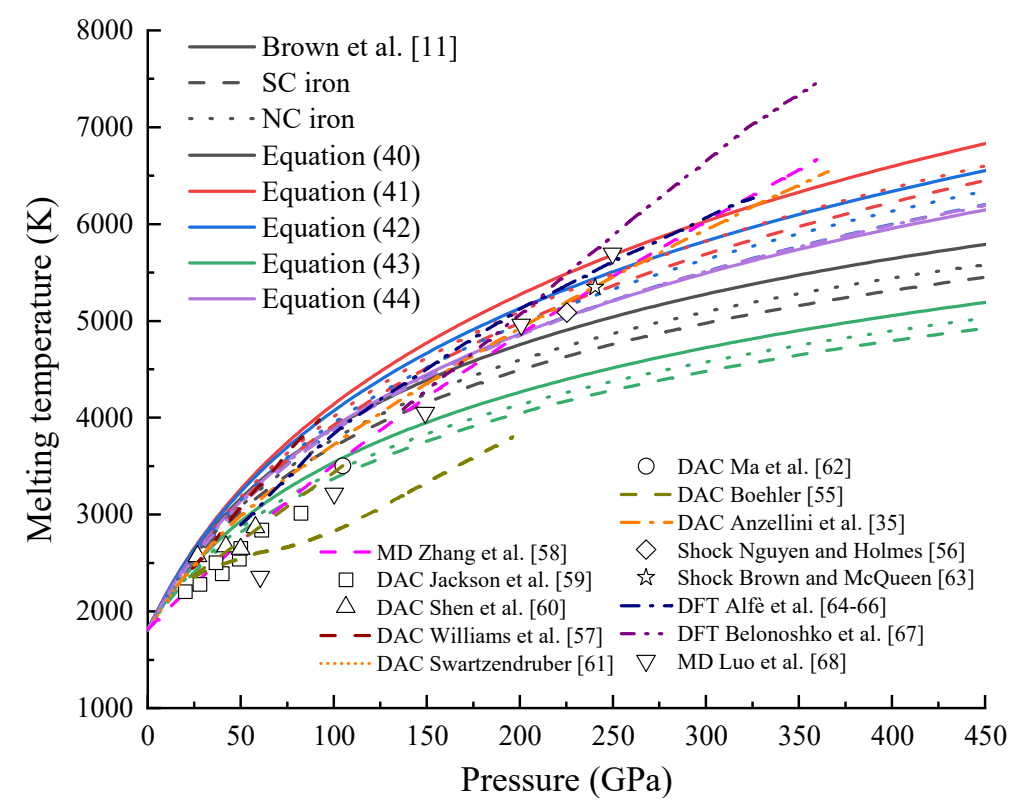

Figure 8. Comparison of the melting temperature of iron from present calculations with previous studies [35,55-68].

As shown in Figure 8, the temperature rises quickly to the melting point because of the adiabatic process under shock compression, and $T_{m}$ shows an increasing trend as the pressure increases. The results from Equations (41), (42) and (44) present a similar and close trend; the red curve at the top corresponds to Equation (41), while the green curve at the bottom corresponds to Equation (43), and the maximum error is $29.5 \%$ at $450 \mathrm{GPa}$. Moreover, the melting temperature in SC iron is a little lower than that in NC iron; the maximum error is $2.5 \%$ at $450 \mathrm{GPa}$.

At lower pressure $(<100 \mathrm{GPa})$, all curves except the green one are very close; this shows that the prediction abilities of Equations (40)-(42) and (44) in the low-compression area are almost the same. It can be found that $T_{m}$ calculated by Equations (40) and (44) agrees well with DAC experimental data in [57], and $T_{m}$ calculated by Equation (43) agrees well with DAC experimental data in $[59,60,62] . T_{m}$ calculated by Equations (41) and (42) is little higher than that obtained from DFT calculations [64-66]. However, the difference between the results gradually increased at higher pressure ( $>100 \mathrm{GPa})$. The increasing trend indicates that in the low-compression area, the effect of $\gamma_{0}$ on $T_{m}$ is insignificant; the effect of $\gamma_{0}$ on $T_{m}$ becomes more significant with increasing pressure. This feature shows the opposite trend compared with the $\gamma(V)$ results. In Figure 8, it can be found that the DAC 
experimental data of Anzellini et al. [35], the theoretical data obtained by MD calculations conducted by Zhang et al. [58], and DFT calculations conducted by Alfè et al. [64-66] are in good agreement. Thus, the orange melting curve obtained by the DAC experiment up to $\sim 350 \mathrm{GPa}$ was chosen as the main reference in this study. The results obtained from Equations (40)-(44) are similar to the orange line, although there are some differences in the results. At lower pressure ( $<100 \mathrm{GPa})$, the black line obtained by Equation (40) is the closest to the orange line, and the green line (Equation (43)) and purple line (Equation (44)) slightly deviate from the orange line. This indicates that Equation (40) can be used to approximately predict $T_{m}$ under $100 \mathrm{GPa}$. In the entire pressure range, the orange line is within the calculation result from Equation (44) at pressure less than $300 \mathrm{GPa}$; the result obtained from Equation (44) is lower than the orange line above $300 \mathrm{GPa}$. This result shows that the calculation results under $300 \mathrm{GPa}$ can cover the experimental data, and the results obtained at higher pressures are lower than the experimental data.

\subsection{Mie-Grüneisen Equation of State}

The Mie-Grüneisen EOS can be obtained from Equations (45) and (46) with the Hugoniot curve (Equation (45)) and the cold curve (Equation (46)) as the reference curves. The Hugoniot curve is obtained via MD simulation. Based on previous analysis, the Grüneisen coefficient $\gamma$ in this study is calculated by Equations (32) and (36). The cold pressure and cold energy are calculated from the Born-Mayer potential. Figure 9 shows the Mie-Grüneisen EOS of iron in 3D contour form of SC iron based on the cold pressure and cold energy, with the Hugoniot relations as the reference.
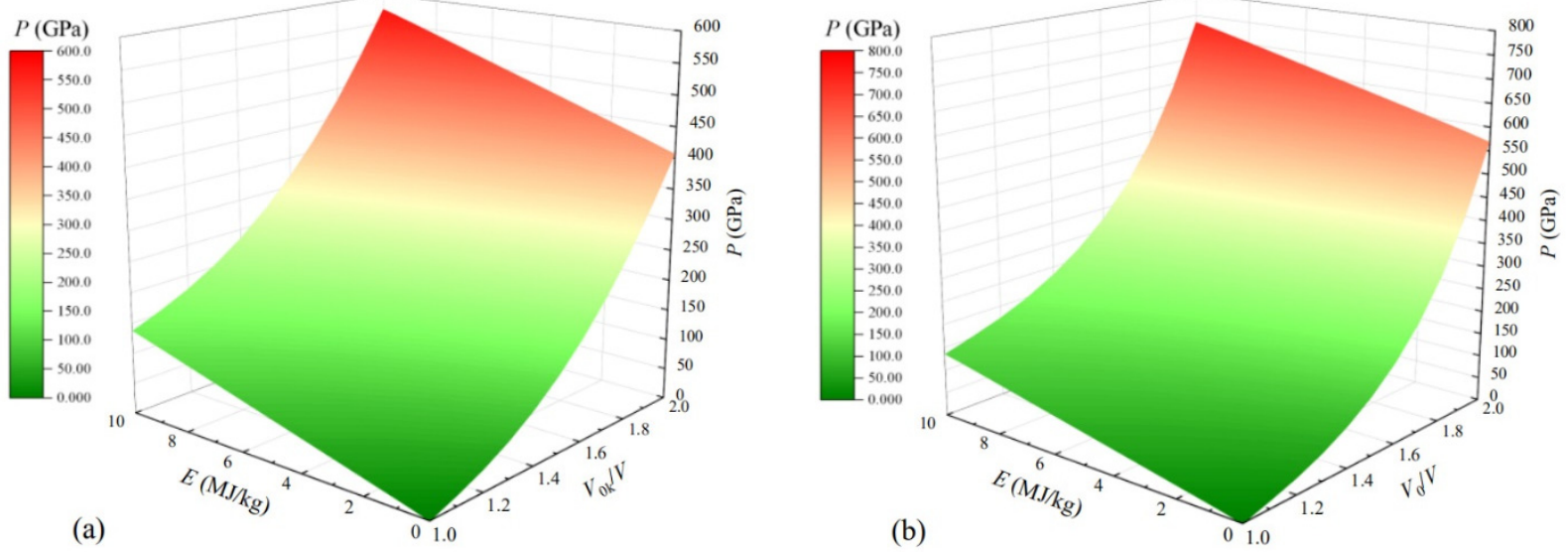

Figure 9. Mie-Grüneisen EOS in P-V-E form with reference from the cold curve (a) and the Hugoniot curve (b).

The Mie-Grüneisen EOS is described in the pressure-specific volume-internal energy $(P-V-E)$ space. In the front view, the whole Mie-Grüneisen EOS space presents a concave surface characteristic, as shown in Figure 9. The maximum pressure of EOS calculated from the cold curve or the Hugoniot curve as the reference will be different. The maximum pressure when the Hugoniot relations are utilized as the reference is $200 \mathrm{GPa}$ higher than that when the cold pressure and cold energy are the references. The results indicate that the reference data have a certain impact on the surface characteristic and values of the Mie-Grüneisen EOS.

\section{Conclusions}

In this study, a micromechanical approach for determining the Mie-Grüneisen EOS parameters of iron under the Hugoniot states was investigated using the MSST method in MD simulation, and the shock Hugoniot, cold curve, melting temperature, and Grüneisen coefficient are also discussed. The simulation results show that the difference between the linear relationships of the shock Huguenot of SC and NC iron was insignificant, and both agree well with the experiment data. Compared with the results at GS $5 \mathrm{~nm}$ and $25 \mathrm{~nm}$, 
the GS effect of iron was not significant at high pressures. The difference between the Born-Mayer potential and the Morse potential in calculating the cold pressure was almost the same as that in calculating the cold energy, but the cold pressure and cold energy in SC iron were a little higher than those in NC iron. As the pressure increased, the Grüneisen coefficient gradually decreased, while the melting temperature gradually increased; the two trends were opposite. The Grüneisen coefficient $\gamma$ is calculated by Equations (16c) and (18c), which have a better performance in this study. The Mie-Grüneisen EOS presented a concave surface characteristic in 3D contour space; the pressure with the Hugoniot curve as the reference was higher than that with the cold curve as the reference, which indicates that different reference curves have a certain impact on the surface characteristic and values of Mie-Grüneisen EOS. The research results in this paper prove the feasibility of the MSST method and provide a reference for studying the EOS of other metals under high pressures. Materials in practical applications are very complex and contain various impurities and defects, and both impurities and defects affect their properties. We examined pure SC iron and perfect NC iron in this study with the MSST method. In future work, we will consider impurities and defects to study the dynamic response characteristics of materials under more complex conditions.

Author Contributions: Conceptualization, investigation and writing-original draft preparation, Y.W.; methodology and software, Y.W. and X.Y.; resources, F.W. and J.D.; supervision, X.Z. and H.C.; project administration and funding acquisition, X.Z. All authors have read and agreed to the published version of the manuscript.

Funding: This research was funded by the foundation of the National Key Defense Laboratory for Computational Physics through the project "Study on the constitutive model of metal materials with damage effect under strong dynamic load", grant number HXo2020-74.

Institutional Review Board Statement: Not applicable.

Informed Consent Statement: Not applicable.

Data Availability Statement: The data that support the findings of this study are available from the corresponding author upon reasonable request.

Acknowledgments: The authors are grateful to Jun Chen of the Institute of Applied Physics and Computational Mathematics Beijing for his valuable suggestions and discussions. We would like to express our sincere appreciation to the anonymous referee for his or her valuable suggestions and corrections.

Conflicts of Interest: The authors declare that we have no known competing financial interest or personal relationships that could have appeared to influence the work reported in this paper.

\section{References}

1. Batani, D.; Morelli, A.; Tomasini, M.; Benuzzi-Mounaix, A.; Philippe, F.; Koenig, M.; Marchet, B.; Masclet, I.; Rabec, M.; Reverdin, C.; et al. Equation of state data for iron at pressures beyond 10 Mbar. Phys. Rev. Lett. 2002, 88, 235502. [CrossRef]

2. Hai-Jun, H.; Fu-Qian, J.; Ling-Cang, C.; Yan, B. Grüneisen Parameter along Hugoniot and Melting Temperature of $\varepsilon$-Iron: A Result from Thermodynamic Calculations. Chin. Phys. Lett. 2005, 22, 836. [CrossRef]

3. Dorogokupets, P.I.; Dymshits, A.M.; Litasov, K.D.; Sokolova, T.S. Thermodynamics and Equations of State of Iron to 350 GPa and 6000 K. Sci. Rep. 2017, 7, 41863. [CrossRef]

4. Joshi, R.H.; Bhatt, N.K.; Thakore, B.Y.; Vyas, P.R.; Jani, A.R. Grüneisen parameter and equations of states for copper-High pressure study. Comput. Condens. Matter 2018, 15, 79-84. [CrossRef]

5. Yang, X.; Zeng, X.; Pu, C.; Chen, W.; Chen, H.; Wang, F. Molecular dynamics modeling of the Hugoniot states of aluminum. AIP Adv. 2018, 8, 105212. [CrossRef]

6. Shao, J.L.; He, A.M.; Wang, P. Atomistic simulations on the dynamic properties of shock and release melting in single crystal Al. Comput. Mater. Sci. 2018, 151, 240-245. [CrossRef]

7. Nagayama, K. Extended Rice-Walsh equation of state for metals based on shock Hugoniot data for porous samples. J. Appl. Phys. 2017, 121, 175902. [CrossRef]

8. Barker, L.M. Alpha-phase Hugoniot of iron. J. Appl. Phys. 1975, 46, 2544-2547. [CrossRef]

9. Thomas, S.A.; Hawkins, M.C.; Matthes, M.K.; Gray, G.T., III; Hixson, R.S. Dynamic strength properties and alpha-phase shock Hugoniot of iron and steel. J. Appl. Phys. 2018, 123, 175902. [CrossRef] 
10. Kadau, K.; Germann, T.C.; Lomdahl, P.S.; Holian, B.L. Microscopic view of structural phase transitions induced by shock waves. Science 2002, 296, 1681-1684. [CrossRef] [PubMed]

11. Brown, J.M.; Fritz, J.N.; Hixson, R.S. Hugoniot data for iron. J. Appl. Phys. 2000, 88, 5496-5498. [CrossRef]

12. Zaretsky, E.B. Shock response of iron between 143 and 1275 K. J. Appl. Phys. 2009, 106, 023510. [CrossRef]

13. Liu, X.; Mashimo, T.; Kawai, N.; Sano, T.; Zhou, X. Isotropic phase transition of single-crystal iron (Fe) under shock compression. J. Appl. Phys. 2018, 124, 215101. [CrossRef]

14. Sjostrom, T.; Crockett, S. Quantum molecular dynamics of warm dense iron and a five-phase equation of state. Phys. Rev. E 2018, 97, 053209. [CrossRef]

15. Kadau, K.; Germann, T.C.; Lomdahl, P.S.; Holian, B.L. Atomistic simulations of shock-induced transformations and their orientation dependence in bcc Fe single crystals. Phys. Rev. B 2005, 72, 064120. [CrossRef]

16. Kadau, K.; Germann, T.C.; Lomdahl, P.S.; Albers, R.C.; Wark, J.S.; Higginbotham, A.; Holian, B.L. Shock waves in polycrystalline iron. Phys. Rev. Lett. 2007, 98, 135701. [CrossRef]

17. Belkheeva, R.K. Equation of state for a highly porous material. High Temp. 2015, 53, 348-357. [CrossRef]

18. Khishchenko, K.V. Equation of state of sodium for modeling of shock-wave processes at high pressures. Math. Montisnigri 2017, 40, 140-147.

19. Maevskii, K.K.; Kinelovskii, S.A. Numerical simulation of thermodynamic parameters of high-porosity copper. Tech. Phys. 2019, 64, 1090-1095. [CrossRef]

20. Hallajisany, M.; Zamani, J.; Albelda Vitoria, J. Numerical and theoretical determination of various materials Hugoniot relations based on the equation of state in high-temperature shock loading. High Press. Res. 2019, 39, 666-690. [CrossRef]

21. Maevskii, K.K.; Kinelovskii, S.A. Modeling of High-Porosity Copper-Based Mixtures under Shock Loading. J. Appl. Mech. Tech. Phys. 2019, 60, 612-619. [CrossRef]

22. Khishchenko, K.V. Equation of state for niobium at high pressures. Math. Montisnigri 2020, 47, 119-123. [CrossRef]

23. Gilev, S.D. Low-parametric equation of state of aluminum. High Temp. 2020, 58, 166-172. [CrossRef]

24. Heuzé, O. General form of the Mie-Grüneisen equation of state. C. R. Mec. 2012, 340, 679-687. [CrossRef]

25. Zhang, X.F.; Qiao, L.; Shi, A.S.; Zhang, J.; Guan, Z.W. A cold energy mixture theory for the equation of state in solid and porous metal mixtures. J. Appl. Phys. 2011, 110, 013506. [CrossRef]

26. Patel, N.N.; Sunder, M. High pressure melting curve of osmium up to 35 GPa. J. Appl. Phys. 2019, 125, 055902. [CrossRef]

27. Slater, J.C. Introduction to Chemical Physics; Chapter XIII; McGraw-Hill: New York, NY, USA, 1939.

28. Dugdale, J.S.; MacDonald, D.K.C. The thermal expansion of solids. Phys. Rev. 1953, 89, 832. [CrossRef]

29. Vashchenko, V.Y.; Zubarev, V.N. Concerning the Grüneisen constant. Fiz. Tverd. Tela 1963, 5, 886-890.

30. Al'tshuler, L.V. Use of shock waves in high-pressure physics. Phys. Uspekhi 1965, 8, 52-91. [CrossRef]

31. Cui, G.; Yu, R. Volume and pressure dependence of Grüneisen parameter $\gamma$ for solids at high temperatures. Phys. B Condens. Matter 2007, 390, 220-224. [CrossRef]

32. Sha, X.; Cohen, R.E. Lattice dynamics and thermodynamics of bcc iron under pressure: First-principles linear response study. Phys. Rev. B 2006, 73, 104303. [CrossRef]

33. Xi-Jun, L.; Xian-Ming, Z.; Fan-Hou, W.; Fu-Qian, J. Restudy of Grüneisen Parameter of Iron in the Pressure Range of 90-160 GPa. Chin. Phys. Lett. 2001, 18, 85. [CrossRef]

34. Errandonea, D. High-pressure melting curves of the transition metals Cu, Ni, Pd, and Pt. Phys. Rev. B 2013, 87, 054108. [CrossRef]

35. Anzellini, S.; Dewaele, A.; Mezouar, M.; Loubeyre, P.; Morard, G. Melting of iron at Earth's inner core boundary based on fast X-ray diffraction. Science 2013, 340, 464-466. [CrossRef]

36. Bouchet, J.; Mazevet, S.; Morard, G.; Guyot, F.; Musella, R. Ab initio equation of state of iron up to 1500 GPa. Phys. Rev. B 2013, 87, 094102. [CrossRef]

37. Barton, M.A.; Stacey, F.D. The Grüneisen parameter at high pressure: A molecular dynamical study. Phys. Earth Planet. Inter. 1985, 39, 167-177. [CrossRef]

38. Maillet, J.B.; Mareschal, M.; Soulard, L.; Ravelo, R.; Lomdahl, P.S.; Germann, T.C.; Holian, B.L. Uniaxial Hugoniostat: A method for atomistic simulations of shocked materials. Phys. Rev. E 2000, 63, 016121. [CrossRef]

39. Vočadlo, L.; Dobson, D.P.; Wood, I.G. An ab initio study of nickel substitution into iron. Earth Planet. Sci. Lett. 2006, $248,147-152$. [CrossRef]

40. Reed, E.J.; Fried, L.E.; Joannopoulos, J.D. A method for tractable dynamical studies of single and double shock compression. Phys. Rev. Lett. 2003, 90, 235503. [CrossRef]

41. Wen, P.; Demaske, B.; Spearot, D.E.; Phillpot, S.R.; Tao, G. Effect of the initial temperature on the shock response of $\mathrm{Cu}_{50} \mathrm{Zr}_{50}$ bulk metallic glass by molecular dynamics simulation. J. Appl. Phys. 2021, 129, 165103. [CrossRef]

42. Dewapriya, M.A.N.; Miller, R.E. Energy absorption mechanisms of nanoscopic multilayer structures under ballistic impact loading. Comput. Mater. Sci. 2021, 195, 110504. [CrossRef]

43. Chen, J.; Chen, W.; Chen, S.; Zhou, G.; Zhang, T. Shock Hugoniot and Mie-Grüneisen EOS of TiAl alloy: A molecular dynamics approach. Comput. Mater. Sci. 2020, 174, 109495. [CrossRef]

44. Born, M.; Mayer, J.E. Zur Gittertheorie der Ionenkristalle. Z. Phys. 1932, 75, 1-18. [CrossRef]

45. Morse, P.M. Diatomic molecules according to the wave mechanics. II. Vibrational levels. Phys. Rev. 1929, 34, 57. [CrossRef]

46. Eliezer, S.; Ghatak, A.; Hora, H. Fundamentals of Equations of State; World Scientific: Singapore, 2002; pp. $197-207$. 
47. Segletes, S.B.; Walters, W.P. On theories of the Grüneisen parameter. J. Phys. Chem. Solids 1998, 59, 425-433. [CrossRef]

48. Jacobs, M.H.G.; Schmid-Fetzer, R. Thermodynamic properties and equation of state of fcc aluminum and bcc iron, derived from a lattice vibrational method. Phys. Chem. Miner. 2010, 37, 721-739. [CrossRef]

49. Burakovsky, L.; Preston, D.L. An analytic model of the Grüneisen parameter all densities. J. Phys. Chem. Solids 2004, 65, 1581-1587. [CrossRef]

50. Ross, M. Generalized Lindemann melting law. Phys. Rev. 1969, 184, 233. [CrossRef]

51. Haynes, W.M. CRC Handbook of Chemistry and Physics; CRC Press: Boca Raton, FL, USA, 2014.

52. Mendelev, M.I.; Han, S.; Srolovitz, D.J.; Ackland, G.J.; Sun, D.Y.; Asta, M. Development of new interatomic potentials appropriate for crystalline and liquid iron. Philos. Mag. 2003, 83, 3977-3994. [CrossRef]

53. Hirel, P. Atomsk: A tool for manipulating and converting atomic data files. Comput. Phys. Commun. 2015, 197, 212-219. [CrossRef]

54. Prieto, F.E.; Renero, C. Equation for the Shock Adiabat. J. Appl. Phys. 1970, 41, 3876-3883. [CrossRef]

55. Boehler, R. Temperatures in the Earth's core from melting-point measurements of iron at high static pressures. Nature 1993, 363, 534-536. [CrossRef]

56. Nguyen, J.H.; Holmes, N.C. Melting of iron at the physical conditions of the Earth's core. Nature 2004, 427, 339-342. [CrossRef] [PubMed]

57. Williams, Q.; Jeanloz, R.; Bass, J.; Svendsen, B.; Ahrens, T.J. The melting curve of iron to 250 gigapascals: A constraint on the temperature at Earth's center. Science 1987, 236, 181-182. [CrossRef]

58. Zhang, W.J.; Liu, Z.Y.; Liu, Z.L.; Cai, L.C. Melting curves and entropy of melting of iron under Earth's core condi-tions. Phys. Earth Planet. Inter. 2015, 244, 69-77. [CrossRef]

59. Jackson, J.M.; Sturhahn, W.; Lerche, M.; Zhao, J.; Toellner, T.S.; Alp, E.E.; Sinogeikin, S.V.; Bass, J.D.; Murphy, C.A.; Wicks, J.K. Melting of compressed iron by monitoring atomic dynamics. Earth Planet. Sci. Lett. 2013, 362, 143-150. [CrossRef]

60. Shen, G.; Prakapenka, V.B.; Rivers, M.L.; Sutton, S.R. Structure of liquid iron at pressures up to 58 GPa. Phys. Rev. Lett. 2004, 92, 185701. [CrossRef]

61. Swartzendruber, L.J. The Fe (Iron) system. Bull. Alloy Phase Diagr. 1982, 3, 161-165. [CrossRef]

62. Ma, Y.; Somayazulu, M.; Shen, G.; Mao, H.K.; Shu, J.; Hemley, R.J. In situ X-ray diffraction studies of iron to Earth-core conditions. Phys. Earth Planet. Inter. 2004, 143-144, 455-467. [CrossRef]

63. Brown, J.M.; McQueen, R.G. Phase transitions, Grüneisen parameter, and elasticity for shocked iron between 77 GPa and 400 GPa. J. Geophys. Res. 1986, 91, 7485-7494. [CrossRef]

64. Alfè, D. Temperature of the inner-core boundary of the Earth: Melting of iron at high pressure from first-principles coexistence simulations. Phys. Rev. B 2009, 79, 060101. [CrossRef]

65. Alfè, D.; Cazorla, C.; Gillan, M.J. The kinetics of homogeneous melting beyond the limit of superheating. J. Chem. Phys. 2011, 135, 024102. [CrossRef] [PubMed]

66. Alfè, D.; Price, G.; Gillan, M. Iron under Earth's core conditions: Liquid-state thermodynamics and high-pressure melting curve from ab initio calculations. Phys. Rev. B 2002, 65, 165118. [CrossRef]

67. Belonoshko, A.B.; Ahuja, R.; Johansson, B. Quasi-ab initio molecular dynamic study of Fe melting. Phys. Rev. Lett. 2000, 84, 3638-3641. [CrossRef]

68. Luo, F.; Cheng, Y.; Chen, X.R.; Cai, L.C.; Jing, F.Q. The melting curves and entropy of iron under high pressure. J. Chem. Eng. Data 2011, 56, 2063-2070. [CrossRef] 